\title{
A modified constraint shifting homotopy method for solving general nonlinear multiobjective programming
}

\author{
Zhichuan Zhu ${ }^{\mathrm{a}, \mathrm{b}, *}$, Yonghong Yaoc \\ a School of Statistics, Jilin University of Finance and Economics, Changchun, Jilin 130117, China. \\ ${ }^{b}$ School of Mathematics and Statistics, Northeast Normal University, Changchun, Jilin 130024, China. \\ ${ }^{c}$ Department of Mathematics, Tianjin Polytechnic University, Tianjin 300387, China.
}

Communicated by N. Shahzad

\begin{abstract}
In this paper, for solving the general nonconvex multiobjective programming with both inequality and equality constraints, a modified constraint shifting homotopy is constructed, and the existence and global convergence of the smooth homotopy pathway is proven for any initial point in the almost Euclidean space under some mild conditions. The advantage of the newly proposed method requires that the initial point can be chosen much more conveniently, which needs to be only in the shifted feasible set not necessarily in the original feasible set. Meanwhile, the normal cone condition for proving the global convergence, which is much weaker than the existing interior method, need only be satisfied at the boundary of the shifted feasible set but not the original constraint set. (C)2017 All rights reserved.
\end{abstract}

Keywords: Homotopy method, nonconvex programming, multiobjective programming, global convergence. 2010 MSC: 90C26, 65H20, 90-08.

\section{Introduction}

Multiobjective programming is a part of mathematical programming dealing with decision problems characterized by multiple and conflicting objective functions which are to be optimized over a feasible set of decisions. Multiobjective programming has been extensively applied in many areas of human activity, such as engineering, management, economics, military, variational problems, and other fields, for more details see, e.g., [2, 4, 7-9, 13, 15-18, 22].

In this paper, we will consider the following general multiobjective programming problem:

$$
\begin{array}{cl}
\min & f(x) \\
\text { s.t. } & g(x) \leqslant 0, \\
& h(x)=0,
\end{array}
$$

where $x \in R^{n}, f(x)=\left(f_{1}(x), f_{2}(x), \ldots, f_{p}(x)\right)^{\top}, g(x)=\left(g_{1}(x), g_{2}(x), \ldots, g_{m}(x)\right)^{\top}$, and $h(x)=\left(h_{1}(x), h_{2}(x)\right.$, $\left.\ldots, h_{l}(x)\right)^{\top}$ are twice continuous differential functions. If the equality constraint $h(x)=0$ is nonlinear, the

\footnotetext{
*Corresponding author

Email addresses: zhuzcnh@126.com (Zhichuan Zhu), yaoyonghong@aliyun.com (Yonghong Yao)
} 
problem (1.1) is nonconvex programming. For convenience, let the sets be denoted as $\Omega=\left\{x \in R^{\mathfrak{n}} \mid g(x) \leqslant\right.$ $0, h(x)=0\}, \Omega^{0}=\left\{x \in R^{\mathfrak{n}} \mid g(x)<0, h(x)=0\right\}$, and $I(x)=\left\{i \in\{1,2, \ldots, m\} \mid g_{i}(x)=0\right\}$.

Among many methods, the following weighed sum method which transforms the problem (1.1) into a single-objective programming is popular and efficient:

$$
\begin{array}{ll}
\min & \lambda^{\top} f(x) \\
\text { s.t. } & g(x) \leqslant 0 \\
& h(x)=0
\end{array}
$$

where $\lambda \in \mathscr{A}=\left\{\lambda \in R_{++}^{p}: \sum_{i=1}^{p} \lambda_{i}=1\right\}, R_{+}^{n}$ and $R_{++}^{n}$ denote the nonnegative and positive orthant of $R^{n}$, respectively.

As we have known that the problem (1.2) is still a nonconvex programming when $h(x)$ is nonlinear and it is difficult to obtain a global optimal solution to the nonconvex programming, even if one wants to obtain a local optimal solution or a solution to the Karush-Kuhn-Tucker (KKT) system of nonconvex programming, it usually needs some much stronger assumptions to obtain its global convergence. As a globally convergent method, the homotopy method has been paid much attention for solving nonlinear problems since 1970's, see, e.g., [3, 6, 20]. Especially, the combined homotopy method, which was proposed and its globally convergence can be proven under much weaker conditions since 1990's for solving nonconvex programming, has been an important tool for numerically solving nonlinear programming, fixed point problem, principal-agent problem, convex multiobjective programming and so on, see, e.g., $[10-12,23,26,30,32,34]$.

In 2008, the combined homotopy interior point method was generalized to solve the general multiobjective programming problem under the so-called normal cone condition and the homotopy was constructed in [21] as follows:

$$
H(w, t)=\left(\begin{array}{c}
(1-t)(\nabla f(x) \lambda+\nabla g(x) y)+\nabla h(x) z+t\left(x-x^{0}\right) \\
Y g(x)-t Y^{0} g\left(x^{0}\right) \\
h(x) \\
(1-t)\left(1-\sum_{i=1}^{p} \lambda_{i}\right) e-t\left(\lambda-\lambda^{0}\right)
\end{array}\right)=0
$$

where $w=(x, \lambda, y, z)^{\top} \in R^{n} \times R_{++}^{p} \times R_{+}^{m} \times R^{l}, x^{0} \in \Omega^{0}, Y=\operatorname{diag}\left(y_{1}, y_{2}, \ldots, y_{m}\right), \lambda^{0}>0, e$ is a vector with elements 1 , and $\sum_{i=1}^{p} \lambda_{i}^{0}=1$. However, the solution of (1.3) must yield $\lambda=\lambda^{0}$ for any $t \in[0,1]$, i.e., $\lambda$ is fixed. In fact, from the last equation of (1.3) and noting $\sum_{i=1}^{p} \lambda_{i}^{0}=1$, we can get $((1-t) p+t)\left(1-\sum_{i=1}^{p} \lambda_{i}\right)=$ 0 . Since $(1-t) p+t \neq 0$ for any $t \in[0,1]$, we have $1-\sum_{i=1}^{p} \lambda_{i}=0$. Hence, $\lambda=\lambda^{0}$ must hold by the last equation of (1.3).

In 2012, to weaken the normal cone condition, a modified homotopy interior point method was presented under the so-called quasinormal cone condition and the modified homotopy is constructed in [27] as follows:

$$
H(w, t)=\left(\begin{array}{c}
(1-t)\left(\nabla f(x) \lambda+\nabla g(x) y+t \eta(x) y^{2}\right)+\nabla h(x) z+t\left(x-x^{0}\right) \\
Y g(x)-t Y^{0} g\left(x^{0}\right) \\
h(x) \\
(1-t)\left(1-\sum_{i=1}^{p} \lambda_{i}\right) e-t\left(\lambda^{5 / 2}-\left(\lambda^{0}\right)^{5 / 2}\right)
\end{array}\right)=0
$$

where $\eta(x)$ is a positive linear independent map with respect to $\nabla g(x)$ and $\nabla h(x)$ for any $x \in \Omega$. But, this method still requires a strictly feasible initial point, so it is not convenient to implement.

To relax the limitation of the strictly feasible initial point, Yu and Shang [26] presented a constraint shifting homotopy method for solving nonlinear programming with only inequality constraints. In 2009, 
Pérez et al. [14] solved homotopy curve tracking optimization problems in the general case of an infeasible starting point through controlling the relaxation of the constraints. In 2014, Jayswal and Choudhury [5] devised an exact $l_{1}$ exponential penalty function method for solving multiobjective optimization problems with exponential-type invexity. In 2015, Zhu and Xiong [28] generalized the constraint shifting homotopy method to solve the nonconvex multiobjective programming problems with only inequality constraints. However, this constraint shifting method for solving nonlinear programming or multiobjective programming with only inequality constraints can not be generalized directly to the general nonlinear programming with both inequality constraint and equality constraint, the counter example can be found in [24].

In this paper, to solve the general multiobjective programming with both inequality constraint and equality constraint, a constraint shifting combined homotopy is constructed and the existence and global convergence of a smooth homotopy path is obtained under some mild conditions. The advantage of the proposed method is that the initial point does not need to be necessarily in the original feasible set but only in the shifted feasible set, and the normal cone condition need only be satisfied in the boundary of the shifted feasible set not the original constraint set.

The remainder of this paper is organized as follows. In Section 2, some assumptions and lemmas will be introduced. In Section 3, a constraint shifting combined homotopy is constructed and the existence and global convergence of a smooth pathway from any given initial point in the shifted feasible set to a KKT point is proven. In Section 4, a conclusion will be given.

\section{Preliminaries}

As it is known that it is difficult to obtain the global solution of nonconvex nonlinear programming, we can only obtain the solution to its KKT systems. Let $x \in \Omega$, we say that $x$ is a KKT point of problem (1.1) if there exists $(\lambda, y, z) \in R_{+}^{n+m} \times R^{l}$ such that:

$$
\nabla f(x) \lambda+\nabla g(x) y+\nabla h(x) z=0, \quad Y g(x)=0, \quad h(x)=0, \quad 1-\sum_{i=1}^{p} \lambda_{i}=0,
$$

where $\nabla f(x)=\left(\nabla f_{1}(x), \nabla f_{2}(x), \ldots, \nabla f_{p}(x)\right) \in R^{n \times p}, \nabla g(x)=\left(\nabla g_{1}(x), \nabla g_{2}(x), \ldots, \nabla g_{m}(x)\right) \in R^{n \times m}$, $\nabla h(x)=\left(\nabla h_{1}(x), \nabla h_{2}(x), \ldots, \nabla h_{l}(x)\right) \in R^{n \times l}$.

Meanwhile, the KKT system of problem (1.1) is (2.1).

Throughout the paper, let $R^{n}, R_{+}^{n}$, and $R_{++}^{n}$ denote $n$-dimensional Euclidean space, nonnegative orthant, and positive orthant of $R^{n}$, respectively. The following parametrized Sard's lemma (see [3, Theorem 2.1]) will be used in this paper. Let $B \subset R^{n}$ be an open set and let $\varphi: B \rightarrow R^{p}$ be a $C^{\alpha}(\alpha>\max \{0, n-p\})$ mapping; we say that $\pi \in R^{p}$ is a regular value of $\varphi$ if

$$
\text { Range }[\partial \varphi(\theta) / \partial \theta]=R^{p}, \quad \forall \theta \in \varphi^{-1}(\pi) .
$$

Lemma 2.1. Let $\mathrm{B} \subset \mathrm{R}^{\mathrm{n}}, \mathrm{D} \subset \mathrm{R}^{\mathrm{m}}$ be open sets, and let $\varphi: \mathrm{B} \times \mathrm{D} \rightarrow \mathrm{R}^{\mathrm{k}}$ be a $\mathrm{C}^{\alpha}$ mapping, where $\alpha>$ $\max \{0, \mathrm{~m}-\mathrm{k}\}$. If $0 \in \mathrm{R}^{\mathrm{k}}$ is a regular value of $\varphi$, then for almost all $\mathrm{b} \in \mathrm{B}, 0$ is a regular value of $\varphi_{\mathrm{b}}=\varphi(\mathrm{b}, \cdot)$.

\section{Main result}

In this paper, for any $t \in[0,1]$, let $\tilde{g}_{i}(x, t)$ and $\tilde{h}_{j}(x, t)=h_{j}(x)-\operatorname{th}\left(x^{0}\right), j=1,2, \ldots, l$ be the shifted constraint functions, which satisfy the following conditions:

(A1) $\tilde{g}_{i}(x, t), i=1,2, \ldots, m$ and $\tilde{h}_{j}(x, t), j=1,2, \ldots, l$ are three times continuous differentiable;

(A2) $\tilde{g}_{i}(x, 0)=g_{i}(x), i=1,2, \ldots, m$ and $\tilde{h}_{j}(x, 0)=h_{j}(x), j=1,2, \ldots, l$. For convenience, we denote $\Omega(t)=\left\{x: \tilde{g}_{i}(x, t) \leqslant 0, \tilde{h}_{j}(x, t)=0, i=1,2, \ldots, m, j=1,2, \ldots, l\right\}, \Omega^{0}(t)=\left\{x: \tilde{g}_{i}(x, t)<0, \tilde{h}_{j}(x, t)=\right.$ 
$0, i=1,2, \ldots, m, j=1,2, \ldots, l\}, \partial \Omega(t)=\Omega(t) \backslash \Omega^{0}(t)$, and $I_{t}(x)=\left\{i \in\{1,2, \ldots, m\}: \tilde{g}_{i}(x, t)=0\right\}$, respectively. let $\nabla \tilde{g}(x, t)$ and $\nabla \tilde{h}(x, t)$ denote the gradient of $\tilde{g}(x, t)$ and $\tilde{h}(x, t)$ with respect to the variate $x$, respectively. To solve the KKT systems (2.1) of problem (1.1), for any randomly chosen $\xi \in R^{n}$ and any given $\eta \in R_{++}^{m}$, a modified constraint shifting homotopy (MCSH) is constructed as follows:

$$
H(w, t)=\left(\begin{array}{c}
(1-t)(\nabla f(x) \lambda+\nabla \tilde{g}(x, t) y)+\nabla \tilde{h}(x, t) z+t\left(x-x^{0}\right)+t(1-t) \xi \\
Y \tilde{g}(x, t)+t \eta \\
\tilde{h}(x, t) \\
(1-t)\left(1-\sum_{i=1}^{p} \lambda_{i}\right) e-t\left(\lambda^{\frac{5}{2}}-\left(\lambda^{0}\right)^{\frac{5}{2}}\right)
\end{array}\right)=0
$$

where $w=(x, \lambda, y, z)^{\top} \in R^{n} \times R_{++}^{p} \times R_{+}^{m} \times R^{l}, x^{0} \in \Omega^{0}(1), Y=\operatorname{diag}\left(y_{1}, y_{2}, \ldots, y_{m}\right)$, the shifted constraint functions $\tilde{g}_{i}(x, t), i=1,2, \ldots, m$ and $\tilde{h}_{j}(x, t), j=1,2, \ldots, l$ also satisfy the following conditions:

(A3) (Slater condition) For all $t \in[0,1], \Omega^{0}(t) \neq \phi$ and $\cup_{t \in[0,1]} \Omega(t)$ is bounded.

(A4) (Positive-linearly independent condition) For all $t \in[0,1], x \in \Omega(t)$, matrix

$$
\left\{\left(\nabla \tilde{g}_{\mathfrak{i}}(x, \mathrm{t})\right)_{i \in \mathrm{I}_{\mathrm{t}}(\mathrm{x})}, \nabla \tilde{\mathrm{h}}(\mathrm{x}, \mathrm{t})\right\}
$$

is positive-linearly independent (see [19]) at $x$.

(A5) (The normal cone condition) For all $x \in \partial \Omega(1)$, the normal cone of $\Omega(1)$ only meets $\partial \Omega(1)$ at $x$, i.e., for all $x \in \partial \Omega(1)$,

$$
\left\{x+\sum_{i \in I_{1}(x)} \nabla \tilde{g}_{i}(x, 1) y_{i}+\sum_{j=1}^{m} \nabla \tilde{h}_{j}(x, 1) z_{j} \mid i \in I_{1}(x), y_{i} \geqslant 0, z_{j} \in R^{m}\right\} \cap \Omega(1)=\{x\} .
$$

Remark 3.1. In comparison with [21,27], the normal cone condition (A5) only requires that the constraint shifting set $\Omega(t)$ holds as $t=1$ but not the whole constraint set $\Omega$, and the initial point requires only in the shifted feasible set but not the original feasible set. Therefore, it is much weaker than that of [21, 27].

For the modified homotopy equation (3.1), when $t=0$, the homotopy equation $\mathrm{H}(w, 0)=0$ turns to the KKT systems (2.1) of problem (1.2), when $t=1$, the homotopy equation $H(w, 1)=0$ has a unique simple solution, which can be proved by the following lemma.

Lemma 3.2. If $\mathrm{H}(w, \mathrm{t})$ is defined as (3.1) and conditions (A1)-(A5) hold, then the homotopy equation $\mathrm{H}(w, 1)=0$ has a unique solution

$$
(x, \lambda, y, z)=\left(x^{0}, \lambda^{0}, y^{0}, z^{0}\right)=\left(x^{0}, \lambda^{0},-\left[\operatorname{diag}\left(\tilde{g}\left(x^{0}, 1\right)\right)\right]^{-1} \eta, 0\right) .
$$

Proof. By the condition (A3), suppose that $(\bar{x}, \bar{\lambda}, \bar{y}, \bar{z})$ is a solution of homotopy equation $\mathrm{H}(w, 1)=0$. From $H((\bar{x}, \bar{\lambda}, \bar{y}, \bar{z}), 1)=0, \eta \in R_{++}^{m}$, and $\bar{y} \geqslant 0$, we get $\bar{x} \in \Omega(1)$. Next, we will prove by contradiction and assume $\bar{x} \neq x^{0}$, which implies that $\bar{z} \neq 0$. By the first equation of $H((\bar{x}, \bar{\lambda}, \bar{y}, \bar{z}), 1)=0$, we have $x^{0}=$ $\bar{x}+\nabla \tilde{h}(\bar{x}, 1) \bar{z}$, which contradicts with the condition (A5). Hence, $\bar{x}=x^{0}$ and $\nabla \tilde{h}(\bar{x}, 1) \bar{z}=\nabla \tilde{h}\left(x^{0}, 1\right) \bar{z}=0$. From the condition (A4), we get $\bar{z}=0$. From the second equation of $H((\bar{x}, \bar{\lambda}, \bar{y}, \bar{z}), 1)=0$, we have $\bar{y}=-\left[\operatorname{diag}\left(\tilde{g}\left(x^{0}, 1\right)\right)\right]^{-1}$. By the forth equation of $H((\bar{x}, \bar{\lambda}, \bar{y}, \bar{z}), 1)=0$, we get $\bar{\lambda}=\lambda^{0}$.

Therefore, we obtain the result.

For a given $w^{0} \in \Omega^{0}(1) \times R_{++}^{p} \times R_{++}^{m} \times R^{l}$, the zero-point set of $H(w, t)$ is denoted as follows:

$$
\mathrm{H}_{w^{0}}^{-1}(0)=\left\{(w, t) \in \Omega^{0}(1) \times \mathrm{R}_{++}^{\mathrm{p}} \times \mathrm{R}_{++}^{\mathrm{m}} \times \mathrm{R}^{\mathrm{l}} \times(0,1]: \mathrm{H}(w, \mathrm{t})=0\right\} .
$$


Theorem 3.3. Suppose that conditions (A1)-(A5) hold, then for almost all $w^{0} \in \Omega^{0}(1) \times R_{++}^{p} \times R_{++}^{m} \times R^{l}$, the zero-point set of the homotopy equation (3.1) contains a smooth curve $\Gamma_{w^{0}} \subset \Omega(1) \times R_{+}^{p} \times R_{+}^{m} \times R^{l} \times(0,1]$ starting from $\left(x^{0}, \lambda^{0}, y^{0}, z^{0}, 1\right)$, which terminates in or approaches to the hyperplane $t=0$. If $\left(x^{*}, \lambda^{*}, y^{*}, z^{*}, 0\right)$ is an ending limit point of $\Gamma_{w^{0}}$, then $w^{*}=\left(x^{*}, \lambda^{*}, y^{*}, z^{*}\right)$ is a solution to KKT systems (2.1).

Proof. Let $\tilde{\mathrm{H}}\left(w, \chi^{0}, \lambda^{0}, \xi, \mathrm{t}\right)$ be the same mapping as $\mathrm{H}(w, \mathrm{t})$ but also taking $\left(\chi^{0}, \lambda^{0}, \xi\right)$ as variate. Considering the submatrix of the Jacobian $\mathrm{D} \tilde{H}\left(w, x^{0}, \lambda^{0}, \xi, \mathrm{t}\right)$ :

$$
\frac{\partial \tilde{\mathrm{H}}\left(w, x^{0}, \lambda^{0}, \xi, \mathrm{t}\right)}{\partial\left(x^{0}, \lambda^{0}, y, \xi\right)}=\left(\begin{array}{cccc}
-\mathrm{t} \mathrm{I}_{\mathrm{n}} & 0 & (1-\mathrm{t}) \nabla \tilde{\mathrm{g}}(\mathrm{x}, \mathrm{t}) & \mathrm{t}(1-\mathrm{t}) \mathrm{I}_{\mathrm{n}} \\
0 & 0 & \operatorname{diag}(\tilde{\mathrm{g}}(x, \mathrm{t})) & 0 \\
\nabla \tilde{\mathrm{h}}\left(x^{0}, \mathrm{t}\right)^{\top} & 0 & 0 & 0 \\
0 & \frac{5}{2} \mathrm{t}\left(\lambda^{0}\right)^{\frac{3}{2}} \mathrm{I}_{\mathrm{p}} & 0 & 0
\end{array}\right) .
$$

For any parameter $t \in(0,1)$, from the vector $\eta \in R_{++}^{m}$ and the second equation $Y \tilde{g}(x, t)+t \eta=0$ of the homotopy equation (3.1), we obtain that the matrix $\operatorname{diag}\left(\tilde{g}_{1}(x, t), \tilde{g}_{2}(x, t), \ldots, \tilde{g}_{m}(x, t)\right)$ is nonsingular. Since for any $x^{0} \in \Omega^{0}(1), \nabla \tilde{h}\left(x^{0}, t\right)$ is a matrix of full column rank, we have that $\nabla \tilde{h}\left(x^{0}, t\right)^{\top}$ is a matrix of full row rank. By the fact that $\lambda^{0} \in R_{++}^{p}$, we can get that $\frac{\partial \tilde{H}\left(w, x^{0}, \lambda^{0}, \xi, t\right)}{\partial\left(x^{0}, \lambda^{0}, y, \xi\right)}$ is a matrix of full row rank for any $t \in(0,1)$, which implies that the matrix $\operatorname{DH}\left(w, x^{0}, \lambda^{0}, \xi, t\right)$ is full row rank for any $t \in(0,1)$.

When the parameter $t=1$, for any $w^{0} \in \Omega^{0}(1) \times R_{++}^{p} \times R_{++}^{m} \times R^{l}$, noting that the matrix

$$
\frac{\partial \mathrm{H}\left(w^{0}, 1\right)}{\partial w}=\left(\begin{array}{cccc}
\mathrm{I} & 0 & 0 & \nabla \tilde{\mathrm{h}}\left(x^{0}, 1\right) \\
\mathrm{Y}^{0} \nabla \tilde{\mathrm{g}}\left(\chi^{0}, 1\right)^{\top} & 0 & \operatorname{diag}\left(\tilde{\mathrm{g}}\left(x^{0}, 1\right)\right) & 0 \\
\nabla \tilde{\mathrm{h}}\left(x^{0}, 1\right)^{\mathrm{T}} & 0 & 0 & 0 \\
0 & -\frac{5}{2}\left(\lambda^{0}\right)^{\frac{3}{2}} \mathrm{I}_{\mathfrak{p}} & 0 & 0
\end{array}\right)
$$

is nonsingular, we obtain that the matrix $\operatorname{DH}\left(w, x^{0}, \lambda^{0}, \xi, t\right)$ is full row rank for any $t \in(0,1]$, which implies that 0 is a regular value of $\tilde{H}\left(w, x^{0}, \lambda^{0}, \xi, t\right)$. Therefore, by Lemma 2.1, for almost all $\left(x^{0}, \lambda^{0}, \xi\right) \in$ $\Omega^{0}(1) \times R_{++}^{p} \times R^{n}, 0$ is a regular value of $H(w, t)$. For any given $w^{0} \in \Omega^{0}(1) \times R_{++}^{p} \times R_{++}^{m} \times R^{l}$, if 0 is a regular value of $\mathrm{H}(w, \mathrm{t})$, by the facts that $\mathrm{H}\left(w^{0}, 1\right)=0$, the matrix $\frac{\partial \mathrm{H}\left(w^{0}, 1\right)}{\partial w}$ is nonsingular, and the famous implicit function theorem, we know that the zero-point set $\mathrm{H}_{w^{0}}^{-1}(0)$ must contain a smooth curve $\Gamma_{w^{0}}$, which starts from $\left(x^{0}, \lambda^{0}, y^{0}, z^{0}, 1\right)$, goes into $\Omega^{0}(1) \times R_{++}^{p} \times R_{++}^{m} \times R^{l} \times(0,1)$, and terminates in the boundary of $\Omega(t) \times R_{++}^{p} \times R_{+}^{m} \times R^{l} \times[0,1]$.

Next, we will prove that the projection of the smooth curve $\Gamma_{w^{0}}$ on the component $\lambda$ is bounded by contradiction. Suppose that the conclusion does not hold, since the interval $(0,1]$ is bounded, there must exist a sequence $\left\{\left(w^{k}, t_{k}\right)\right\} \subset \Gamma_{w^{0}}$ such that $t_{k} \rightarrow t_{*},\left\|\lambda^{k}\right\| \rightarrow \infty$. By the forth equation of (3.1), we have that

$$
\left(\begin{array}{c}
1-t_{k} \\
1-t_{k} \\
\vdots \\
1-t_{k}
\end{array}\right)-\left(\begin{array}{c}
\left(1-t_{k}\right) \lambda_{1}^{k}+\left(1-t_{k}\right) \sum_{i \neq 1} \lambda_{i}^{k}+t_{k}\left(\lambda_{1}^{k}\right)^{5 / 2} \\
\left(1-t_{k}\right) \lambda_{2}^{k}+\left(1-t_{k}\right) \sum_{i \neq 2}^{5} \lambda_{i}^{k}+t_{k}\left(\lambda_{2}^{k}\right)^{5 / 2} \\
\vdots \\
\left(1-t_{k}\right) \lambda_{p}^{k}+\left(1-t_{k}\right) \sum_{i \neq p} \lambda_{i}^{k}+t_{k}\left(\lambda_{p}^{k}\right)^{5 / 2}
\end{array}\right)-\left(\begin{array}{c}
t_{k}\left(\lambda_{1}^{0}\right)^{5 / 2} \\
t_{k}\left(\lambda_{2}^{0}\right)^{5 / 2} \\
\vdots \\
t_{k}\left(\lambda_{p}^{0}\right)^{5 / 2}
\end{array}\right)=0 .
$$

If we assume that $\left\|\lambda^{k}\right\| \rightarrow \infty$ as $k \rightarrow \infty$, it implies that the set $\left\{i \in\{1,2, \ldots, p\}: \lim _{k \rightarrow \infty} \lambda_{i}^{k}=\infty\right\} \neq \emptyset$. Then, by the fact that $t_{k} \rightarrow t_{*}$ and $\lambda^{k}>0$, we have that the second part in the left-hand side of some equations in (3.2) will tend to infinity as $k \rightarrow \infty$. But, the first part and the third part are bounded, which imply that the equation (3.2) is impossible. Thus, the component $\lambda$ is bounded.

Let $\left(x^{*}, \lambda^{*}, y^{*}, z^{*}, t^{*}\right)$ be a limit point of $\Gamma_{w^{0}}$ when $t \rightarrow 0$. Only the following five cases are possible: 
(i) $\left(x^{*}, \lambda^{*}, y^{*}, z^{*}, t^{*}\right) \in \Omega(1) \times R_{+}^{p} \times R_{+}^{m} \times R^{l}, t^{*}=1,\left\|\left(y^{*}, z^{*}\right)\right\|<\infty$;

(ii) $\left(x^{*}, \lambda^{*}, y^{*}, z^{*}, t^{*}\right) \in \Omega\left(t^{*}\right) \times R_{+}^{p} \times R_{+}^{m} \times R^{l}, t^{*} \in[0,1],\left\|\left(y^{*}, z^{*}\right)\right\|=\infty$;

(iii) $\left(x^{*}, \lambda^{*}, y^{*}, z^{*}, t^{*}\right) \in \Omega\left(t^{*}\right) \times R_{+}^{p} \times R_{+}^{m} \times R^{l}, t^{*} \in(0,1),\left\|\left(y^{*}, z^{*}\right)\right\|<\infty$;

(iv) $\left(x^{*}, \lambda^{*}, y^{*}, z^{*}, t^{*}\right) \in \partial \Omega\left(t^{*}\right) \times R_{+}^{p} \times R_{++}^{m} \times R^{l}, t^{*} \in(0,1),\left\|\left(y^{*}, z^{*}\right)\right\|<\infty$;

(v) $\left(x^{*}, \lambda^{*}, y^{*}, z^{*}, t^{*}\right) \in \Omega \times R_{+}^{p} \times R_{+}^{m} \times R^{l}, t^{*}=0,\left\|\left(y^{*}, z^{*}\right)\right\|<\infty$.

From Lemma 3.2, we know that the point $w^{0}=\left(x^{0}, \lambda^{0}, y^{0}, z^{0}\right)$ is the unique simple solution of $\mathrm{H}\left(w^{0}, 1\right)=0$, which implies that case (i) is impossible.

If the case (ii) happens, there must exist a sequence of points $\left\{\left(x^{k}, \lambda^{k}, y^{k}, z^{k}, t^{k}\right)\right\} \subset \Gamma_{w^{0}}$ such that $t^{k} \rightarrow t^{*} \in[0,1], \lambda^{k} \rightarrow \lambda^{*}, x^{k} \rightarrow \theta^{*} \in \Omega\left(t^{*}\right)$, and $\left\|\left(y^{k}, z^{k}\right)\right\| \rightarrow \infty$ as $k \rightarrow \infty$. From the first equation of (3.1), we have

$$
\left(1-t^{k}\right)\left(\nabla f\left(x^{k}\right) \lambda^{k}+\sum_{i=1}^{m} y_{i}^{k} \nabla \tilde{g}_{i}\left(x^{k}, t^{k}\right)\right)+\sum_{j=1}^{l} \nabla \tilde{h}_{j}\left(x^{k}, t^{k}\right) z_{j}^{k}+t^{k}\left(x^{k}-x^{0}\right)+t^{k}\left(1-t^{k}\right) \xi=0 .
$$

Then, as $k$ tends to infinity, only the following two subcases are possible: (I) $t^{*}=1 ;$ (II) $t^{*} \in[0,1)$.

(I) $t^{*}=1$. If $\left\|\left(\left(1-t^{k}\right) y^{k}, z^{k}\right)\right\|<\infty$, without loss of generality, suppose that $\left(\left(1-t^{k}\right) y^{k}, z^{k}\right) \rightarrow\left(y^{*}, z^{*}\right)$, then $y_{i}^{*}=0$ for $i \notin I_{1}\left(\theta^{*}\right)$ from the second equation of (3.1). Taking limits in (3.3) as $k \rightarrow \infty$, we can obtain

$$
\begin{aligned}
x^{0} & =x^{*}+\lim _{k \rightarrow \infty}\left[\left(1-t^{k}\right)\left(\nabla f\left(x^{k}\right) \lambda^{k}+\sum_{i=1}^{m} \nabla \tilde{g}_{i}\left(x^{k}, t^{k}\right) y_{i}^{k}\right)+\sum_{j=1}^{m} \nabla \tilde{h}_{j}\left(x^{k}, t^{k}\right) z_{j}^{k}\right] \\
& =x^{*}+\sum_{j=1}^{m} \nabla \tilde{h}_{j}\left(x^{*}, 1\right) z_{j}^{*}+\lim _{k \rightarrow \infty} \sum_{i \in I_{1}\left(x^{*}\right)}\left(1-t^{k}\right) y_{i}^{k} \nabla \tilde{g}_{i}\left(x^{k}, t^{k}\right) \\
& =x^{*}+\sum_{j=1}^{m} \nabla \tilde{h}_{j}\left(x^{*}, 1\right) z_{j}^{*}+\sum_{i \in I_{1}\left(x^{*}\right)} y_{i}^{*} \nabla \tilde{g}_{i}\left(x^{*}, 1\right)
\end{aligned}
$$

which contradicts with condition (A5).

If $\left\|\left(\left(1-t^{k}\right) y^{k}, z^{k}\right)\right\| \rightarrow \infty$, the discussion is the same as case (II).

(II) $t^{*} \in[0,1)$. Without loss of generality, suppose that $\left(\left(1-t^{k}\right) y^{k}, z^{k}\right) /\left\|\left(\left(1-t^{k}\right) y^{k}, z^{k}\right)\right\| \rightarrow\left(\alpha^{*}, \beta^{*}\right)$ with $\left\|\left(\alpha^{*}, \beta^{*}\right)\right\|=1$ and $\alpha_{i}^{*}=0$ for $i \notin I_{t^{*}}\left(\theta^{*}\right)$. Dividing the both sides of equation (3.3) by $\left\|\left(\left(1-t^{k}\right) y^{k}, z^{k}\right)\right\|$ and taking limits as $k \rightarrow \infty$, we have

$$
\sum_{i \in \mathrm{I}_{\mathrm{t}^{*}}\left(x^{*}\right)} \alpha_{i}^{*} \nabla \tilde{g}_{i}\left(x^{*}, \mathrm{t}^{*}\right)+\sum_{j=1}^{m} \beta_{j}^{*} \nabla \tilde{h}_{j}\left(x^{*}, t^{*}\right)=0,
$$

which contradicts with the condition (A4).

From the above discussions (I) and (II), we conclude that case (ii) is also impossible.

From $\operatorname{diag}\left(\tilde{\mathrm{g}}\left(x^{*}, \mathrm{t}^{*}\right)\right) \mathrm{y}^{*}+\mathrm{t}^{*} \eta=0$, we have that $\mathrm{t}^{*}>0$ and $\mathrm{y}^{*} \in \partial \mathrm{R}_{+}^{\mathrm{m}}$, i.e., $\mathrm{y}_{i}^{*}=0$ for some $1 \leqslant$ $i \leqslant m$ can not happen simultaneously. Therefore, case (iii) is impossible. If $y^{*}>0$ and $t^{*}>0$, from $\operatorname{diag}\left(\tilde{\mathrm{g}}\left(x^{*}, \mathrm{t}^{*}\right)\right) \mathrm{y}^{*}+\mathrm{t}^{*} \eta=0$, we get $\operatorname{diag}\left(\tilde{\mathrm{g}}\left(\mathrm{x}^{*}, \mathrm{t}^{*}\right)\right)<0$, which implies that case (iv) is impossible.

As a conclusion, case $(\mathrm{v})$ is the only possible case. That is $\Gamma_{w^{0}}$ must terminate in or approach to the hyperplane at $t^{*}=0$ and $w^{*}=\left(x^{*}, \lambda^{*}, y^{*}, z^{*}\right)$ is a solution to the KKT systems (2.1).

\section{Conclusions}

In this paper, a new homotopy equation for solving the general multiobjective programming is constructed and the global convergence has been proven under much weaker conditions. For the MCSH 
method, the standard predictor-corrector procedure is usually implemented to numerically trace the homotopy path $\Gamma_{w^{0}}$. The computational steps are usually taken by the following three steps: firstly, computing the tangent direction for the first predictor step; secondly, computing secant directions for the midway predictor steps; finally, using Newton iterations for solving an augmented system for the corrector steps. Many results and discussions for the predictor-corrector algorithms and the convergence have appeared, see e.g., $[1,3,24,31,33]$.

Therefore, the detailed algorithm is omitted here. Our contribution in the paper is theoretical and the numerical simulations on performance for the MCSH can be implemented as reference [28].

\section{Acknowledgment}

This paper was supported by the Jilin Provincial Science and Technology Development Plan funded project (20170520050JH), the China Postdoctoral Science Foundation funded project (No. 2016M591468), the National Natural Science Foundation of China (No. 11601174), and the Key Program of Jilin University of Finance and Economics (No. 2016Z10).

\section{References}

[1] E. L. Allgower, K. Georg, Numerical Continuation Methods: An Introduction, Springer, Berlin, (1990). 4

[2] T. Antczak, A. Pitea, Parametric approach to multitime multiobjective fractional variational problems under ( $F, \rho)$ convexity, Optim. Control Appl. Meth., 37 (2016), 831-847. 1

[3] S. N. Chow, J. Mallet-Paret, J. A. Yorke, Finding zeros of maps: homotopy methods that are constructive with probability one, Math. Comp., 32 (1978), 887-899. 1, 2, 4

[4] G. Isac, M. M. Kosteva, M. M. Wiecek, Multiple-objective appproximation of feasible but unsolvable linear complementarity problems, J. Optim. Theory Appl., 86 (1995), 389-405. 1

[5] A. Jayswal, S. Choudhury, An exact $l_{1}$ exponential penalty function method for multiobjective optimization problems with exponential-type invexity, J. Oper. Res. Soc. China, 2 (2014), 75-91. 1

[6] R. B. Kellogg, T. Y. Li, J. Yorke,A constructive proof the Brouwer fixed-point theorem and computational results, SIAM J. Numer. Anal., 13 (1976), 473-483. 1

[7] H. Konno, T. Kuno, Linear multiplicative programming, Math. Programming, 56 (1992), 51-64. 1

[8] T. C. Koopmans, Activity Analysis of Production and Allocation, Wiley, New York, (1951).

[9] M. M. Kosterva, M. M. Wiecek, Linear complementarity problems and multiple objective programming, Math. Programming, 60 (1993), 349-359. 1

[11] Z.-H. Lin, B. Yu, G.-C. Feng, A combined homotopy interior point method for convex nonlinear programming, Appl. Math. Comput., 84 (1997), 193-211.

[12] Z. H. Lin, D. L. Zhu, Z. P. Sheng, Finding a minimal efficient solution of a convex multiobjective program, J. Optim. Theory Appl., 118 (2003), 587-600. 1

[10] Q. H. Liu, Z. H. Lin, Homotopy interior point method of solving weak efficient solutions for multiobjective programming problems, Acta Math. Appl. Sin. Engl. Ser., 23 (2000), 188-195. 1

[13] V. Pareto, Course d'Economic Politique, Librairie Droz, Switzerland, (1964). 1

[14] V. M. Perez, J. E. Renaud, L. T. Watson, Homotopy curve tracking in approximate interior point optimization, Optim. Eng., 10 (2009), 91-108. 1

[15] A. Pitea, T. Antczak, Proper efficiency and duality for a new class of nonconvex multitime multiobjective variational problems, J. Inequal. Appl., 2014 (2014), 20 pages. 1

[16] A. Pitea, M. Postolache, Duality theorems for a new class of multitime multiobjective variational problems, J. Global Optim., 54 (2012), 47-58.

[17] A. Pitea, M. Postolache, Minimization of vectors of curvilinear functionals on the second order jet bundle Necessary conditions, Optim. Lett., 6 (2012), 459-470.

[18] A. Pitea, M. Postolache, Minimization of vectors of curvilinear functionals on the second order jet bundle Sufficient efficiency conditions, Optim. Lett., 6 (2012), 1657-1669. 1

[19] L. Qi, Z.-X. Wei, On the constant positive linear dependence condition and its application to SQP methods, SIAM J. Optim., 10 (2000), 963-981. 3

[20] S. Smale, A convergent process of price adjustment and global newton methods, J. Math. Econom., 3 (1976), 107-120. 1

[21] W. Song, G. M. Yao, Homotopy Method for a General Multiobjective Programming Problem, J. Optim. Theory Appl., 138 (2008), 139-153. 1, 3.1

[22] J. Von Neumann, O. Morgenstern, Theory of Games and Economic Behavior, Princeton University Press, New Jersey, (1944). 1 
[23] Y. H. Yang, X. R. Lu, Q. H. Liu, Infeasible interior-point homotopy method for non-convex programming under normal cone condition, J. Jilin Univ. Sci., 45 (2007), 365-368. 1

[24] L. Yang, B. Yu, Q. Xu, A constraint shifting homotopy method for general non-linear programming, Optimization, 63 (2014), 585-600. 1, 4

[26] B. Yu, Y. Shang, Boundary moving combined homotopy method for nonconvex nonlinear programming, J. Math. Res. Expos., 26 (2006), 831-834. 1, 1

[27] X. Zhao, S. G. Zhang, Q. H. Liu, Homotopy Interior-Point Method for a General Multiobjective Programming Problem, J. Appl. Math., 2012 (2012), 12 pages. 1, 3.1

[28] Z.-C. Zhu, H.-J. Xiong, A constraint shifting homotopy method for finding a minimal efficient solution of nonconvex multiobjective programming, Optim. Methods Softw., 30 (2015), 634-642. 1, 4

[30] Z.-C. Zhu, B. Yu, A modified homotopy method for solving the principal-agent bilevel programming problem, Comput. Appl. Math., 2016 (2016), 26 pages. 1

[31] Z.-C. Zhu, B. Yu, Globally convergent homotopy algorithm for solving the KKT systems to the principal agent bilevel programming, Optim. Methods Softw., 32 (2017), 69-85. 4

[32] Z.-C. Zhu, B. Yu, Y.-F. Shang, A modified homotopy method for solving nonconvex fixed points problems, Fixed Point Theory, 14 (2013), 531-544. 1

[33] Z.-C. Zhu, B. Yu, L. Yang, Globally convergent homotopy method for designing piecewise linear deterministic contractual function, J. Ind. Manag. Optim., 10 (2014), 717-741. 4

[34] Z. Zhu, Z. Zhou, Y.-C. Liou, Y.-H. Yao, Y. Xing, A globally convergent method for computing fixed point of self-mapping on general nonconvex set, J. Nonlinear Convex Anal., 18 (2017), 1067-1078. 1 